\title{
1 Estimating the Biological Validity of the DSM for \\ 2 Attention Deficit/Hyperactivity Disorder Using \\ 3 Multivariate Analysis for Small Samples
}

5 Dimitri M. Abramov ${ }^{1}$, Evelyne Vigneau ${ }^{2}$, Saint-Clair Gomes-Junior ${ }^{3}$, Carlos Alberto 6 Mourão-Júnior $^{4}$, Monique Castro-Pontes ${ }^{1}$, Carla Quero Cunha ${ }^{1}$, Leonardo C.

7 deAzevedo ${ }^{1}$, Vladimir V. Lazarev ${ }^{1}$

8 1. Laboratory of Neurobiology and Clinical Neurophysiology, National Institute of 9 Women, Children, and Adolescents' Health Fernandes Figueira, Oswaldo Cruz 10 Foundation, Rio de Janeiro, Brazil;

2. StatSC, Oniris, INRA, Nantes, France;

3.Clinical Research Unit, National Institute of Women, Children, and Adolescents Health

Fernandes Figueira, Oswaldo Cruz Foundation, Rio de Janeiro, Brazil; and

14

4.Laboratory of Psychophysiology, Institute of Biological Sciences, Federal University of Juiz de Fora, Juiz de Fora, Brazil.

\section{Corresponding Author:}

Dimitri Marques Abramov

National Institute of Women, Children, and Adolescents Health Fernandes Figueira, Oswaldo Cruz Foundation Avenida Ruy Barbosa, 716, Flamengo, Rio de Janeiro, RJ

Postal Code: $22.250-020$

Phone: +55 21 2554-1955, +55 21 2554-1805

E-mail: dimitri.abramov@iff.fiocruz.br

Keywords: Attention Deficit/Hyperactivity Disorder, DSM, diagnostic validity, Attention Network Test, event-related potentials 
bioRxiv preprint doi: https://doi.org/10.1101/126433; this version posted September 28, 2018. The copyright holder for this preprint (which was not certified by peer review) is the author/funder. All rights reserved. No reuse allowed without permission.

26 Short Title: Estimating DSM validity for $A D H D$ diagnosis.

Dimitri M. Abramov, 2 


\section{Abstract}

Background. Psychiatric nosology lacks objective biological foundation, as well as typical biomarkers for diagnoses, which raises questions about its validity. The problem is particularly evident concerning Attention Deficit/Hyperactivity Disorder (ADHD). The objective of this study is to estimate whether the "Diagnostic and Statistical Manual of Mental Disorders" (DSM) is biologically valid for ADHD diagnosis using a multivariate analysis for small samples from a large dataset concerning neurophysiological, behavioral, and psychological variables.

Methods: Twenty typically developing boys and 19 boys diagnosed with ADHD, aged 10-13 years, were examined using the Attentional Network Test (ANT) with records of event-related potentials (ERPs). From 815 variables, a reduced number of latent variables (LVs) were extracted with a clustering method, for further reclassification of subjects using the k-means method. This approach allowed multivariate analysis to be applied to a significantly larger number of variables than the number of cases $(E$. Wigneau et al., 2003, 2015)

Results: From datasets including ERPs from the mid-frontal, mid-parietal, right frontal, 4 and central channels, only seven subjects were miss-reclassified by the LVs. An 


\section{Introduction} related to $A D H D$.

estimated specificity of $75.00 \%$ and sensitivity of $89.47 \%$ for DSM were found in the reclassification. The kappa index between DSM and behavioral/psychological/neurophysiological data was 0.75 , which is regarded as a "substantial level of agreement".

Discussion: Results showed that CLV is a useful method for diagnostic classification using a large dataset of small samples, suggesting the biological validity of DSM for ADHD diagnosis, in accordance to alterations in fronto-striatal networks previously (American Psychiatric Association, 1994 - 2013) [1,2] is usually questioned due to its subjective criteria and to the absence of objective tests to define nosological entities [3, 4]. Therefore, the lack of objective determinants makes room for criticism and raises doubts about the nosological criteria itself [4]. This is particularly important in controversial mental diseases such as the Attentional Deficit/Hyperactivity Disorder -

ADHD $[5,6]$. Similar to some other disorders, ADHD diagnosis is based on the 1 quantification of normal behavior characteristics using DSM scores. Thus, DSM has a 2 taxonomy of categories that are defined by dimensional phenomena based on a 
63

64

variation from normality $[7,8]$. Correlations between biological alterations and ADHD are still not enough to support the diagnosis $[8,9]$.

Mental disorders are multidimensional complex entities that can be mostly associated with a pool of biological and neurobehavioral variables, often with non-linear relationships $[8,10]$, rather than being defined by a single distinct biomarker such as an antibody or aberrant protein [11]. This formally determines their complexity; a complex nosological entity such as ADHD may be defined by a large set of different quantitative variables. From this standpoint, multivariate factor analysis techniques have been applied to electroencephalographic or biochemical data since the 1970's in order to develop high-sensitivity and accuracy models for the diagnosis of mental disorders [1216]. However, in order to explain these complex mental conditions in terms of biological correlates, a substantially larger sample of subjects than those set of variables is required. Thus, high-dimensional datasets from relatively few observations or subjects usually appear to be statistically questionable and hamper unambiguous interpretation.

7 In order to tackle this problem (the curse of the dimensionality), approaches based on feature selection in a supervised context or on feature extraction in explorative data analysis (for instance, Principal Components Analysis, Canonical Correlation Analysis) 80 have been investigated. Particularly, a strategy of unsupervised "clustering of variables 81 around latent variables" (CLV) was used, which makes it possible to extract 82 synthetic/latent variables through cluster analyses [17-19]. These few latent variables 
represent dataset informativeness for a classifier methodology in a subsequent step (e.g. inputs for a k-means clustering algorithm). This was the first attempt to apply the CLV method to a large psychophysiological dataset. This method is expected to help solve an important methodological problem of multidimensional approaches in clinical research when recruiting large groups of patients is difficult.

The present study is an attempt to discuss whether DSM is valid for an accurate diagnosis of the nosological entity called ADHD, analyzing a small sample of subjects with well-controlled confounding variables and bias. Large datasets of (1) behavioral variables, (2) neurophysiological data (event-related potentials - ERPs, including late cognitive component, P3 or P300 [20,21]), both obtained while subjects performed the Attention Network Test - ANT [22,23], as well as (3) psychological characteristics (from WISC-III) have been collected from typically developing boys (TD) and boys diagnosed with ADHD using the DSM-IV-TR criteria.

The relationships between ADHD manifestations, evaluated using DSM-IV-TR, and the objective characteristics obtained by the above-mentioned experimental measurements were here analyzed. The aim was to discuss the taxonomic (i.e. classificatory) aspects of ADHD diagnosis using DSM, compared with a data-driven statistical approach, without considering the role of these characteristics in putative brain mechanisms underlying ADHD. This study does not address controversial issues, e.g. if the ADHD phenomenology actually constitutes a mental disorder or not. The present 
103 study seeks to discuss the validity of the DSM as a tool to identify this phenomenology,

104 making inferences about its sensitivity and specificity.

\section{Methods}

\section{Design and Subject Selection}

This transversal and exploratory study was conducted in accordance with the

108 Declaration of Helsinki and approved by the Ethics Committee of the National Institute of

109 Women, Children, and Adolescents Health Fernandes Figueira (CAAE

110 08340212.5.0000.5269). All the participants provided their oral consent in the presence

111 of their caregivers, who provided written informed consent after receiving a complete

112 description of the study.

Sixty boys, aged 10-13 years, were included according to DSM-IV-TR: 35 with ADHD (coded as T001 to T035) and 25 TD (coded as C001 to C025). The exclusion

115 criteria were: (1) history of chronic diseases, and any suspicion of psychiatric disorders

116 other than ADHD (psychosis, affective, obsessive-compulsive and tic disorders, phobic

117 and post-traumatic stress conditions, anorexia, bulimia, encopresis, or enuresis) as

118 screened by K-SADS-PL [23]; (2) use of any psychotropic medicines for at least 30 
119 days; (3) estimated I.Q. equal or lower than 80; and (4) less than 6 hours of regular

120 sleep and (5) report of somnolence before the ANT testing.

The following were considered as confounding variables: years in school, monthly

122 family income (in Brazilian Reals), sleep hours the night before, and mean weekly time

123 which the boy dedicated to computer activities and videogames (ranked as follows: 1,

124 less than 2h/week; 2, 2-4h; 3, 5-8h; 5, more than $15 \mathrm{~h} /$ week), learning rate (see below)

125 in the experimental procedure, as well as age.

After applying the exclusion criteria, the behavioral analysis of accuracy (AC) and

127 speed-accuracy tradeoff (see below), and after excluding confounding outliers, 19 ADHD

128 and 20 TD boys remained in the sample, and their data were considered as

129 experimental results. caregivers were shown the DSM-IV-TR criteria and were instructed to point out carefully

133 whether or not each specific criterion was an exact characteristic of their child's

134 behavior. If there was any doubt about or hesitation in any item, it was disregarded.

135 Thus, subjects were classified in accordance with the DSM-IV-TR. 
137 subtests of the Wechsler Intelligence Scale for Children, WISC-III [24, 25]. The

138 Arithmetic and Digit Spam subtests were also performed for the pool of variables used in

139 the multivariate analysis.

\section{Experimental Procedures}

The ANT version adapted for children was used $[20,26]$. A forced, two-choice test was performed, where the subject was instructed to observe the horizontal orientation of

143 a target stimulus (yellow fish), which was (or not) preceded by a cue signal (a red star).

144 The target appeared above or below the fixation point. There were three equiprobable

145 cue conditions corresponding to this signal's position or to its non-appearance: 1) at the

146 subsequent upper or lower position of the target - spatial cue condition; 2) at the central

147 fixation point - neutral cue condition; or 3) no cue condition. The subject had to promptly

148 press the left or right arrow key on the keyboard, according to the horizontal orientation

149 of the target. The test was organized in 8 test blocks, with 24 trials each, and one 150 preceding training block. Reaction time (RT) and accuracy (AC, rate of hits) were 151 recorded.

152 Subjects with AC lower than $70 \%$ and speed-accuracy tradeoff (estimated as AC $153 \times \mathrm{RT})$ lower than the mean sample value minus two standard deviations were excluded. 


\section{EEG Acquisition}

155

156

157

158

159

160

161

162

163

164

165

166

167

168

169

170

171

During the ANT, the subject's EEG was recorded using a Nihon Kohden NK1200

EEG System at 20 scalp points according to the International 10/20 System (Figure 1), with reference at the lateral central leads (linked C3 and C4, the physical reference of the System). Impedance was below $10 \mathrm{k} \Omega$, sampling rate was $1000 \mathrm{~Hz}$ with a 16-bit resolution, and the filters were as follows: low-pass $0.5 \mathrm{~Hz}$, high-pass $100 \mathrm{~Hz}$, and notch $60 \mathrm{~Hz}$.

Figure 1. Electrical potentials related to Attention Network Test. (A) Cue- and target-related potentials and variation in interstimulus voltage during Attention Network Test performance (averaged over all ANT conditions and all subjects). Overview of the ERPs for all channels, including 'C3 minus C4' (normalized amplitudes, $\mu \mathrm{V}$ ), with waves of interest (in dotted squares) to calculate the mean square of the total area under each wave and peak amplitudes/latencies. In the grey box, C and T stand for cue and target onsets, and the trigger signal is identified by dotted lines. Above the ERP graphs, scalp areas are marked with letters with even (right), odd (left) or z (midline) indices: occipital - O, parietal - P, central - C, frontal - F, frontopolar - Fp, mid-temporal - T, posterior temporal - T5 and T6, and anterior temporal - F7 and F8. Scalp scheme for 10-20 montage at the bottom left. (B) ERPs for selected channels, in different colors, showing wave amplitudes $(\mu \mathrm{V})$.

\section{Data Analysis}

Dimitri M. Abramov, 10 
172 Predictor (i.e. DSM score for ADHD as the sum for inattention and

173 hyperactivity/impulsivity scores) and confounding variables were compared between the

174 groups using Student's t-test, after normality and heteroscedasticity assessments were

175 performed using the Shapiro-Wilk and Levene's tests, respectively (Table 1). 
176 Table 1. Predictor and Confounding Variables between Groups

\begin{tabular}{|c|c|c|c|c|c|c|}
\hline & \multicolumn{2}{|c|}{$\begin{array}{c}\text { Typically } \\
\text { Developing } \\
(n=20)\end{array}$} & \multicolumn{2}{|c|}{$\begin{array}{l}\text { ADHD } \\
(n=19)\end{array}$} & \multirow[b]{2}{*}{ T-value $^{\mathrm{a}}$} & \multirow[b]{2}{*}{$p$-value } \\
\hline & $M$ & $S D$ & $M$ & $S D$ & & \\
\hline \multicolumn{7}{|c|}{ Predictor Variables (DSM scores) } \\
\hline Inattention & 2.40 & 1.66 & 7.21 & 1.27 & -10.09 & $<0.001$ \\
\hline Hyperactive+Impulsive & 2.60 & 1.63 & 4.36 & 2.90 & -2.36 & 0.023 \\
\hline Total & 5.00 & 2.75 & 11.63 & 3.00 & -7.19 & $<0.001$ \\
\hline \multicolumn{7}{|l|}{ Confounding Variables } \\
\hline Age (years) & 11.30 & 0.86 & 11.52 & 1.07 & -0.73 & 471 \\
\hline $\begin{array}{l}\text { Estimated I.Q. } \\
\text { Hours of Sleep (previous }\end{array}$ & 109.35 & 13.53 & 97.36 & 12.53 & 2.86 & 0.006 \\
\hline night) & 7.25 & 2.12 & 7.68 & 1.73 & -0.70 & 0.490 \\
\hline Videogame $^{b}$ & 3.40 & 1.23 & 2.89 & 1.66 & 1.08 & 0.286 \\
\hline Computer handling ${ }^{c}$ & 3.30 & 1.26 & 2.94 & 1.68 & 0.74 & 0.462 \\
\hline Years in school & 6.05 & 1.14 & 6.10 & 1.32 & -0.14 & 0.889 \\
\hline Family Incomes $^{d}$ & 6355.00 & 5643.57 & 3978.94 & 4436.91 & 1.46 & 0.153 \\
\hline Learning Index ${ }^{e}$ & 1.22 & 0.34 & 1.26 & 0.30 & -0.36 & 0.721 \\
\hline
\end{tabular}

177

178

179

180

181

182

183

184

185

186

187

188

189

190

${ }^{a}$ Inference using Student's t-test for independent samples, significant differences $(p$ value $<0.05)$ in bold.

${ }^{b, c}$ Videogame and computer handling were scored as: $1=$ less than $2 \mathrm{~h} /$ week; $2=2-4 \mathrm{~h} / \mathrm{wk}, 3=5-8 \mathrm{~h} / \mathrm{wk}, 4$ $=8-15 \mathrm{~h} / \mathrm{wk}, 5=$ more than $15 \mathrm{~h} / \mathrm{wk}$.

${ }^{d}$ in Brazilian Reals $(R \$)$, per month

${ }^{\text {e }}$ Learning Index in the ANT: reaction time (RT) (ms) of last trial block / mean RT (ms) of the first experimental blocks.

Several behavioral variables were gathered from the ANT for each subject: mean AC of task performance, i.e. the percentage of correct responses; mean Reaction Time (RT) and its standard deviation, called intra-individual variation of RT (IVRT), for each cue and target condition. Additionally, data included the ANT scores of the subjects: alerting, orienting, and conflict resolution, mean RT for correct and incorrect responses, and learning rates (the mean scores of the first ANT trial block divided by those of the last experimental block evaluated for RTs and IVRTs). 
192 (see dotted squares in Figure 1 and their measures in Table S1) for each subject, in peak amplitude, (2) peak latency, and (3) the total amplitude under the wave (i.e. the 195 sum of all amplitude values, proportional to the mean amplitude). These three time intervals included late ERPs evoked by 1) the cue, 2) the target stimuli, and 3) the

197 voltage variation between these two ERPs. The same ERP parameters were calculated

198 for the difference between signals of the two central channels (C3 minus C4) instead of 199 estimating the absolute signal values in each of these leads, observing the EEG 200 reference used here $(\mathrm{C} 3+\mathrm{C} 4)$.

201 Thus, the total number of variables of interest (VOI) was 774: four scores obtained from the above-mentioned WISC-III subtests, 29 behavioral variables obtained 203 from ANT, and 741 neurophysiological variables (39 for each of the 18 channels and C3204 C4).

206 regional (local) cerebral activity, while the variables from ANT and WISC provide the 207 integral characteristics of the subject. For the analysis, the activity in certain brain 208 regions of the subjects performing ANT (but not all) was assumed to best correlate with 209 ADHD phenomenology. For this reason, 40 different sets of channels (Table 2) were 210 used for data mining, in an attempt to find the best agreement with DSM (if any). 
211 Table 2. Agreement with DSM (Number of Subjects) by Channel Set and Number of Latent Vectors for 212 Variables of Interest

\begin{tabular}{|c|c|c|c|c|c|c|c|c|c|}
\hline \multirow{2}{*}{ Channel Sets $^{a}$} & \multicolumn{4}{|c|}{ ERPs variables only } & \multicolumn{4}{|c|}{ All variables of Interest } & \multirow{2}{*}{ MEAN } \\
\hline & $1 \mathrm{LV}$ & $2 \mathrm{LV}$ & $3 \mathrm{LV}$ & $4 \mathrm{LV}$ & $1 \mathrm{LV}$ & $2 \mathrm{LV}$ & $3 \mathrm{LV}$ & $4 \mathrm{LV}$ & \\
\hline All Channels & 20 & 24 & 24 & 25 & 20 & 24 & 24 & 28 & 23.63 \\
\hline $\begin{array}{llll}F z & C z & P z & O z\end{array}$ & 23 & 25 & 23 & 22 & 27 & 28 & 29 & 29 & 25.75 \\
\hline $\begin{array}{cccccc}F 7 & T 3 & T 5 & F p 1 & F 3 & C 3-C 4 \\
& P 3 & 01 & F z & P z & \end{array}$ & 21 & 22 & 22 & 24 & 20 & 22 & 27 & 28 & 23.25 \\
\hline $\begin{array}{c}C 3-C 4 \quad F 8 \quad \text { T4 } \quad \text { T6 } F p 2 \quad F 4 \\
\text { P4 O2 } \quad F z \quad P z\end{array}$ & 24 & 24 & 24 & 24 & 24 & 24 & 24 & 24 & 24.00 \\
\hline $\begin{array}{ccccccc}F 7 & \text { T3 } & \text { T5 } & F 8 & \text { T4 } & \text { T6 } & F z \\
C Z & P z & & & \end{array}$ & 20 & 25 & 25 & 24 & 23 & 25 & 29 & 28 & 24.88 \\
\hline $\begin{array}{ccccccc}F 7 & T 3 & T 5 & C 3-C 4 & F 8 & T 4 \\
\text { T6 } & F z & C Z & P Z & \end{array}$ & 22 & 20 & 22 & 24 & 22 & 28 & 28 & 28 & 24.25 \\
\hline $\begin{array}{llllll}F 7 & T 3 & T 5 & F p 1 & C 3-C 4 & F 8 \\
T 4 & T 6 & F p 2 & F z & C z & P z\end{array}$ & 21 & 23 & 24 & 24 & 23 & 28 & 25 & 28 & 24.50 \\
\hline C3-C4 Fz CZ Pz & 27 & 27 & 27 & 26 & 30 & 30 & 30 & 30 & 28.38 \\
\hline$F 7 \quad$ T3 $\quad$ T5 $\quad$ C3-C4 Fz $\quad P z$ & 22 & 28 & 23 & 22 & 22 & 30 & 28 & 29 & 25.50 \\
\hline C3-C4 F8 T4 T6 Fz $P z$ & 20 & 25 & 24 & 22 & 31 & 31 & 29 & 28 & 26.25 \\
\hline F7 $\quad$ T3 $\quad$ C3-C4 Fz $P z$ & 23 & 27 & 20 & 31 & 26 & 29 & 31 & 29 & 27.00 \\
\hline C3-C4 F8 $\quad$ T4 $\quad F z \quad P z$ & 31 & 30 & 28 & 29 & 30 & 30 & 31 & 28 & 29.63 \\
\hline C3-C4 Fz Pz & 30 & 30 & 29 & 30 & 29 & 28 & 30 & 29 & 29.38 \\
\hline 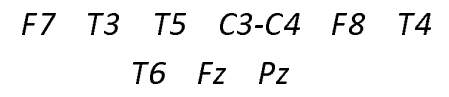 & 21 & 20 & 23 & 22 & 22 & 26 & 28 & 29 & 23.88 \\
\hline$F 7 \quad C 3-C 4 \quad F 8 \quad F z \quad P z$ & 23 & 29 & 29 & 27 & 30 & 28 & 31 & 29 & 28.25 \\
\hline $\begin{array}{llllll}F 7 & F 3 & F 8 & F 4 & F z & P z\end{array}$ & 27 & 25 & 31 & 30 & 29 & 29 & 30 & 31 & 29.00 \\
\hline $\begin{array}{ccccc}F 7 & F 3 \quad C 3-C 4 & F 8 & F 4 & F z \\
P z & & & \end{array}$ & 27 & 27 & 28 & 30 & 28 & 30 & 31 & 30 & 28.88 \\
\hline $\begin{array}{lllll}F 7 & F 3 & F 8 & F 4 & F z\end{array}$ & 25 & 26 & 27 & 27 & 27 & 29 & 29 & 28 & 27.25 \\
\hline $\begin{array}{lllllll}F 7 & F 3 & F 8 & T 4 & F 4 & F z & P z\end{array}$ & 28 & 25 & 29 & 29 & 28 & 29 & 30 & 29 & 28.38 \\
\hline$F 7 \quad F 3 \quad C 3-C 4 \quad F 8 \quad F 4 \quad F z$ & 27 & 26 & 23 & 28 & 27 & 29 & 29 & 28 & 27.13 \\
\hline $\begin{array}{c}F 7 \quad F p 1 \quad F 3 \quad C 3-C 4 \quad F 8 \\
F p 2 \quad F 4 \quad F z \quad P z\end{array}$ & 27 & 29 & 29 & 30 & 28 & 29 & 29 & 26 & 28.38 \\
\hline $\begin{array}{c}F 7 \quad F p 1 \quad F 3 \quad F 8 \quad F p 2 \quad F 4 \\
F z \quad P z\end{array}$ & 29 & 29 & 29 & 29 & 28 & 29 & 29 & 28 & 28.75 \\
\hline 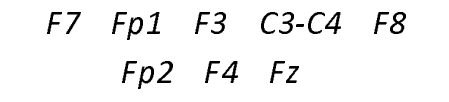 & 28 & 28 & 28 & 28 & 28 & 28 & 27 & 28 & 27.88 \\
\hline$F 7 \quad F p 1 \quad F 3 \quad F 8 \quad F p 2 \quad F 4$ & 27 & 28 & 28 & 28 & 28 & 28 & 28 & 29 & 28.00 \\
\hline
\end{tabular}

Dimitri M. Abramov, 14 


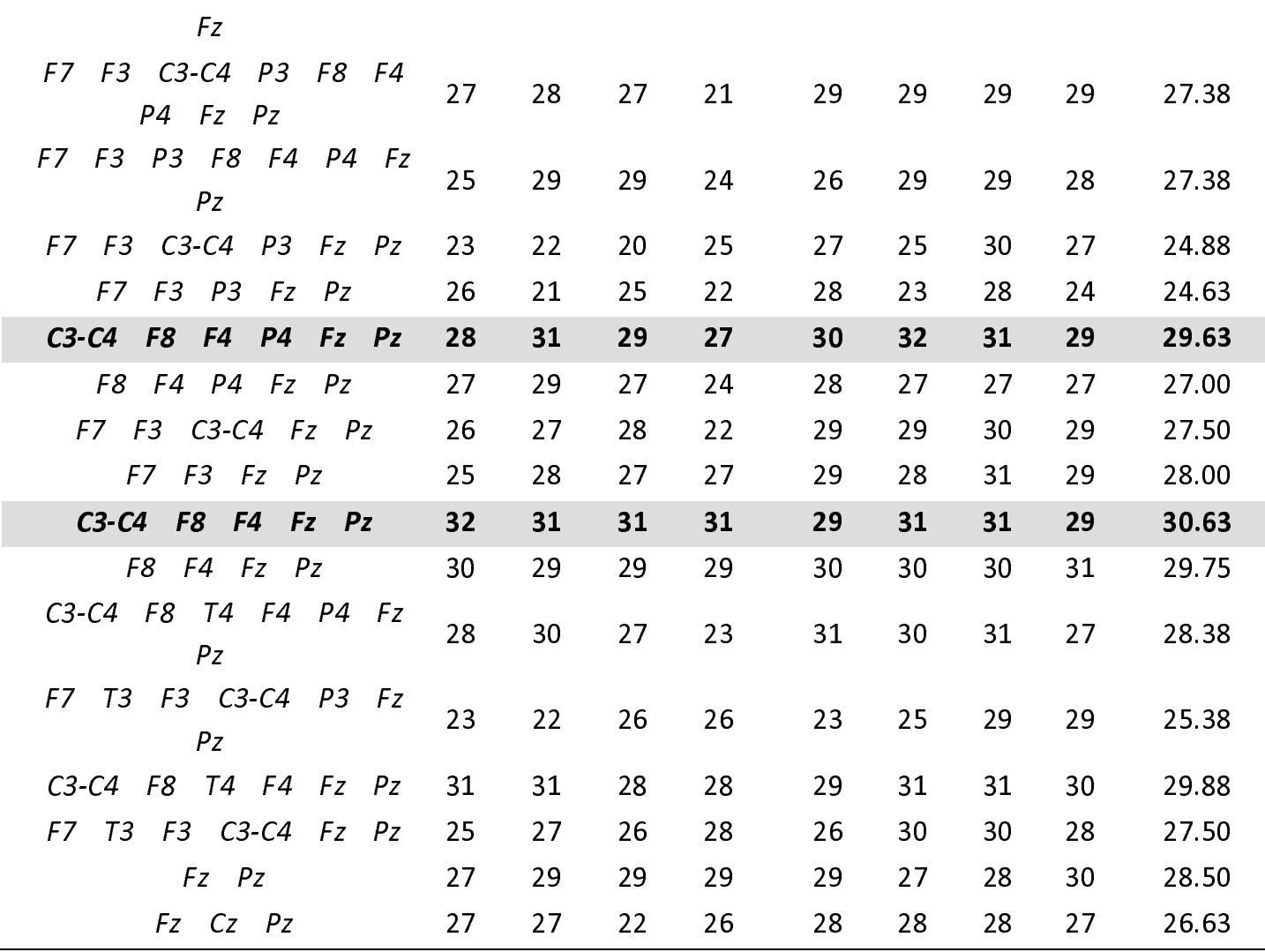

213 a Channel sets according to the International 10-20 system for EEG leads; C3-C4, the channel of difference between 214 two leads.

215 In bold and shaded in gray, higher agreement with DSM.

216 ERPs = event-related potentials; $L V=$ latent variables.

The data from each investigated set of EEG channels were analyzed either with

218 or without behavioral (ANT) and psychological (WISC) variables. They were submitted to

219 an unsupervised method called Clustering of Variables around Latent Variables (CLV)

$220[17,18]$. The aim was to reduce the number of dimensions in the variables space by

221 extracting latent variables (LVs), each of them associated with a cluster of the VOI

222 variables. This clustering approach is based on correlation similarity indices between 
223 variables and aims to identify directional clusters of variables. As it is, each extracted

224 LV, associated with a specific cluster of variables, is defined as the first (standardized)

225 principal component of the variables assigned to that cluster [21]. In our study, the

226 clusters of variables around one to four LVs were systematically considered and the best

227 number of LVs was determined according to the classification of subjects.

228 In fact, our ultimate purpose was to reindex the subjects into two groups

229 according to the objective behavioral, psychological, and neurophysiological data, all of

230 which were summarized around the extracted LVs. A k-means clustering method [27]

231 was performed on the subjects using the LVs. Each reclassification obtained (named

232 "output") was checked for agreement compared to the classification of subjects using the

233 DSM-IV-TR (called “DSM agreement"). The probability that agreement with DSM could

234 be observed under randomness was checked using the Chi-Square test for categorical

235 variables with one degree of freedom, and the expected value was 20 (absolute

236 randomness). The kappa index [28] was also evaluated to estimate the agreement level

237 with DSM (S2 method).

238 In order to determine whether reclassification was consistent, this study analyzed

239 which subjects were reclassified into another group other than his/her original DSM

240 group ("miss-reclassification"). This was performed for each specified agreement rate

241 with DSM, based on the outputs that provided this level of agreement. A measure of 
242 "classifier power" was suggested by dividing the level of agreement with DSM and the

243 number of miss-reclassified subjects in any output with that agreement with DSM.

244 Subsequently, the outputs with the highest classifier power were considered the 245 most representative to estimate the validity of the DSM. Among all outputs, these were 246 submitted to cross-validation using the Leave-One-Out (LOO) strategy, where the 247 consistency of agreement with DSM versus LV was observed across the mean values of 248 the 39 LOO outputs, with 38 subjects each one. The output with the highest consistency 249 of agreement with DSM was regarded as the most representative.

250 Using the most representative outputs, a sensitivity and specificity analysis was 251 suggested for DSM criteria for ADHD.

252 The dataset with variables used in the multivariate analysis is available in the 253 supplementary material (S3 Dataset).

\section{Results}

\section{Evaluating predictor and confounding variables}

256 Control and ADHD groups showed significant differences in the quantitative 257 scores of DSM-IV-TR $(p<0.05)$ (Table 1). The significance level of the difference 258 between the two groups was higher for the inattention score than for the 
259 hyperactivity/impulsivity score. Among variables considered as confounders, the I.Q.

260 scores in ADHD group were lower than in TD boys $(p=0.006)$ although they were

261 always higher than 80. All other variables, including learning rate, were not significantly

262 different between the two groups according to the Student's t-test (normality and

263 homoscedasticity were not statistically rejected).

264 Overview of Event-Related Potentials

Cue and target-related potentials with late peak latency (> 200ms, corresponding

266 to parietal P3) were observed in all channels. Peak amplitude varied from the maximum

267 value of $18 \mu \mathrm{V}$ for the frontopolar target ERP to the minimum value of $1 \mu \mathrm{V}$ for the 268 difference between C3 and C4 (Fig 1).

269 Data Mining and Reclassification of subjects $0.00, p=1.000$ ) to 0.82 (32 subjects, $\chi^{2}=7.20, p=0.004$ ) were found for all 40 channel

272 sets and LV sets (extracting 1 to 4 LVs), with or without behavioral variables enclosed

273 (Table 2). The worst mean agreement level between DSM and K-means reclassification

274 was 23.25 for [F7, T3, T5, Fp1, F3, C3-C4, P3, O1, Fz, Pz] channel set $\left(\chi^{2}=0.43, p=\right.$ 275 0.528); and the best mean agreement level with DSM was 30.62 for [C3-C4, F8, F4, Fz, 276 Pz] channel set $\left(\chi^{2}=5.63, p=0.010\right)$. 
278 C4, F8, F4, Fz, Pz] (using 1 LV from ERP variables alone) and [C3-C4, F8, F4, P4, Fz, 279 Pz] (using 3 LVs from ERP and behavioral variables together). Table 2 shows all the observed agreement rates for all channel sets.

As expected, there was an inverse relationship between the agreements with DSM (from 20 to 32 , according to the trial) and the total number of miss-reclassified subjects (Fig 2). The highest agreement rate found (32 subjects) corresponded to the highest classifier power (4.00).

285

286

287

Figure 2. Classifier Power of the MVA analysis. Number of miss-reclassified subjects per agreement with DSM (number of subjects) in all 320 outputs, and the corresponding classifier power (numbers at the graph marks). See methods.

Regarding an agreement level equal to 32 , the LOO cross-validation showed that only the output from the [C3-C4, F8, F4, Fz, Pz] channel set (using 1 LV from ERP variables alone) was valid, and therefore, it was the most representative: the mean agreement of all 39 outputs was 31.56 subjects (the highest mean agreement among all others, see S4 Output), while the output from [C3-C4, F8, F4, P4, Fz, Pz] (using 3 LVs from ERP and behavioral variables together) had a mean agreement equal to 29.87 
294 subjects. The S4 Output has the agreement data of all outputs and their respective LOO 295 cross-validations (mean values of all 39 outputs).

Seven subjects in the selected output ([C3-C4, F8, F4, Fz, Pz] channel set, using

2971 LV from ERP variables alone) were miss-reclassified: C005, C009, C012, C020, C023

298 (control subjects miss-reclassified to the ADHD group) and T010, T026 (ADHD subjects

299 miss-reclassified to the Control group). Based on these results, the estimated specificity

300 and sensitivity of the DSM-IVTR regarding biological variables were $75.00 \%$ and

$30189.47 \%$, respectively, with accuracy of $82.05 \%$ corresponding to the kappa index of

302 0.75, i.e. a substantial strength of agreement [28].

\section{Discussion}

305 Our study tackled the possibility of examining attention deficit/hyperactivity 306 disorder using the DSM manual, whose criteria seemed to be biologically justified for 307 ADHD diagnosis with "substantial strength of agreement" regarding a specific pool of 308 behavioral, psychological, and neurophysiological variables. A larger source of 309 behavioral and neurophysiological data related to ADHD phenomenology was found in 
310 the ANT, since this test is suitable to access the three dimensions of attention in the

311 same setting [22, 29].

In order to classify subjects as normal or ADHD according to DSM criteria, caregivers were asked which criteria were well-defined characteristics of their children.

314 Other assessment methodologies should also be used along with DSM for ADHD 315 diagnosis both in scientific research and in clinical practice. Moreover, the manual 316 should not be applied as a questionnaire in clinical practice. However, in this case, the

317 object of evaluation is the DSM itself; it was thus the only clinical diagnostic tool used for 318 the classification of subjects as either ADHD or not-ADHD. It was the most objective and controlled way to obtain the scores without interference from any other clinical impression. Supporting the present methodology, the ASRS (the WHO questionnaire for adult ADHD screening) was self-administered [30].

The most informative neurophysiological variables in this pool proved to be

323 topographically asymmetrical and represented the binded information from ERPs in the

324 three time intervals of interest from mid-frontal, mid-parietal, right frontal, and right

325 anterior temporal sites (Fz, Pz, F4, and F8 channels), as well as early ERP components 326 (45 to $290 \mathrm{~ms}$ after target onset) from the 'C3 minus C4' channel. This asymmetry has

327 already been shown to correlate with DSM scores, mainly with hyperactivity/impulsivity

328 [31]. It was not the focus of the present investigation to establish models for ADHD or its

329 neural mechanisms. However, our results revealed certain biological aspects of ADHD 
331 studies have shown brain asymmetries related to ADHD phenomenology, especially in

332 the frontal-striatal network [33-35]. Asymmetrical topographic patterns were shown in our previous studies by spectral and coherence analyses of the resting EEG, with signs of relative inactivation of the frontal and left temporal cortex, known to be responsible for voluntary attention, and which are impaired in ADHD patients [36]. These observations could partially explain the leading role of ERP data from the right frontal regions in discriminating between patients and controls in the present study.

Our results suggest that DSM-IV-TR is actually an effective and biologically justified tool for ADHD diagnosis. Although a Kappa index of 0.75 should be considered

340 with reservations for some clinical settings, a clinical method such as DSM, with sensitivity of $89.47 \%$ and specificity of $75 \%$ with regard to objective behavioral, psychological, and neurophysiological measures, should be considered as particularly

343 relevant considering the complexity of mental disorders, especially those with

344 dimensional clinical phenomenology and without any validated biomarkers, so much so

345 that the only psychiatric tool for diagnosis and intervention is still phenomenological

346 examination, which must be systematically (although qualitatively) performed based on

347 manuals such as the DSM. Furthermore, the ADHD has been regarded as a

348 heterogeneous condition with inattentive, hyperactive, and combined subtypes, which

349 could be the answer for this level of agreement since these subtypes were not predicted 
350 for reclassification. The existence of three discrete subtypes could lead to a flawed 351 reclassification.

The study was designed with a relatively small sample size. Any subject with the 353 slightest suspicion of comorbidity was excluded. Potentially confounding factors

354

355 commonly ignored were monitored (such as manipulating technologies that could lead to the development of specific skills that would interfere with test performance and socioeconomic niche that could determine psychological development). Therefore, the sample should be regarded as well consistent with and representative of ADHD. On the other hand, in order to classify the subjects according to their mental condition (a multidimensional phenomenological complex) it would seem reasonable to gather a considerable number of objective variables (as many as possible). Brain processing during the ANT test as well as ADHD neurophysiology and its behavioral correlates in this study were considered as potentially relevant features requiring further investigation.

Since it did not seem feasible to collect a sample with thousands of subjects evaluating hundreds of variables in an experiment with optimal control of confounders and bias, the dataset had to have a much smaller number of observations than variables. A small but strictly controlled and well-exploited sample was considered preferable, even in conditions of high prevalence such as the ADHD. Multivariate statistical modeling, such as Linear Regression, Discriminant Analysis, or Factor Analysis, are well known as unsuitable for this situation. Thus, in order to tackle a frequently encountered 
370 methodological problem, often referred to as the curse of the dimensionality, an

371 explorative data analysis suitable for small samples was adopted to identify a small

372 number of synthetic latent variables. Instead of the Principal Component Analysis, which

373 does not always provide easily interpretable latent variables (the principal components),

374 an approach with clustering of variables was chosen to reduce data dimensionality to a

375 small number of new latent variables. The CLV method $[17,18]$ has already been applied

376 to a wide range of research domains among which are pattern analysis [37],

377 chemometrics [38], image analysis [39], and analysis scorecards in the health sector

378 [40]. The results seemed promising for optimizing the experimental designs of clinical

379 studies in which it is difficult to recruit large groups of patients.

380 The estimated I.Q. scores were different between the groups diagnosed using

381 DSM-IV-TR, but this was not regarded as bias. Literature has shown that intelligence

382 tests are sensitive to ADHD, and patient scores are generally lower than controls [41].

383 Even taking into account the above restrictions, there is no doubt that the data obtained

384 seem to be quite consistent.

A major limitation of this study is the impossibility of testing all possible combinations among variables. Since the consistency of our paradigm lies on the fact

387 that no biological / behavioral assumptions were considered in the grouping of variables,

388 it is necessary to test them randomly grouped. However, there are more than one million

389 possible combinations of EEG channels. The number of combinations grows 
exponentially if the other variables are included individually. For computational

391 feasibility, sets of variables were arbitrarily chosen for analysis. Considering the eighty

392 sets studied, it can be assumed that DSM has at least a "substantial level of agreement"

393 with biological determinants. Perhaps some untested set of variables may manifest

394 higher agreement with the DSM, but the results found already satisfy our objective of

395 testing the validity of the DSM criteria for ADHD.

The aim of the present study was to draw attention to psychiatric approaches

397 based on diverse and multiple factors intervening in the biological mechanisms of

398 human behavior and its disorders. Therefore, we have found that there is a consistent complex of biological information, which is in accordance with previous knowledge on

400 ADHD, that confers biological validity for DSM criteria for ADHD.

\section{Aknowledgements}

402 We would like to thank The Child's Neurology program of the National Institute of

403 Women, Children, and Adolescents' Health Fernandes Figueira. We are also grateful to

404 Mrs. Maria S. Santana and Mrs. Aldenys Perez for the technical support. Our team is

405 especially grateful to our friend, colleague, and co-author Dr. Adailton Tadeu Pontes, 
406 children's neurologist, who greatly inspired this article, and passed away during its 407 preparation.

\section{References}

409 1. American Psychiatric Association. Diagnostic and statistical manual of mental

410 disorders. 4th ed. Washington, DC: Author; 1994.

411 2. American Psychiatric Association. Diagnostic and statistical manual of mental

412 disorders. 5th ed. Arlington, VA: American Psychiatric Publishing; 2013.

413 3. Insel T. Director's Blog: Transforming Diagnosis. 2013 Apr 29 [cited 06 Jul 2018] In:

414 The National Institute of Mental Health Website. [about 2 screens] Available from:

415 http://www.nimh.nih.gov/about/director/2013/transforming-diagnosis.shtml.

416 4. Wakefield JC. DSM-5, psychiatric epidemiology and the false positives problem.

417 Epidemiol Psychiatr Sci. 2015 Jun;24(3):188-96. doi: 10.1017/S2045796015000116.

418 5. Rafalovich A. Exploring clinician uncertainty in the diagnosis and treatment of

419 attention deficit hyperactivity disorder.Sociol Health IIIn. 2005 Apr;27(3):305-23.

420 6. Strauss V. An ADHD controversy in the mental health community. 2013 February 13

421 [cited 06 July 2018]. In: The Washington Post [Internet]. Available from: 
422 https://www.washingtonpost.com/blogs/answer-sheet/post/an-adhd-controversy-in-the-

423 mental-health-community/2012/02/12/glQAHJun9Q blog.html.

424 7.Maser JD, Akiskal HS. Spectrum concepts in major mental disorders. Psychiatr Clin

425 North Am. 2002 Dec;25(4):xi-xiii.

426 8.Kirmayer LJ, Crafa D. What kind of science for psychiatry? Front Hum Neurosci. 2014

427 Jun 20;8:435. doi: 10.3389/fnhum.2014.00435.

428 9.Furman LM. Attention-deficit hyperactivity disorder (ADHD): does new research

429 support old concepts? J Child Neurol. 2008 Jul;23(7):775-84. doi:

$430 \quad 10.1177 / 0883073808318059$.

431 10. Freitas-Silva LR, Ortega F. Biological determination of mental disorders: a

432 discussion based on recent hypotheses from neuroscience. Cad Saude Publica. 2016

433 Aug 29;32(8):e00168115. doi: 10.1590/0102-311X00168115.

434 11.Scarr E, Millan MJ, Bahn S, Bertolino A, Turck CW, Kapur S, Möller HJ, Dean B.

435 Biomarkers for Psychiatry: The Journey from Fantasy to Fact, a Report of the 2013

436 CINP Think Tank. Int J Neuropsychopharmacol. 2015 Apr 21;18(10):pyv042. doi:

437 10.1093/ijnp/pyv042.

438 12. Bochkarev VK, Lazarev VV, Nikiforov AI, Paniushkina SV, Severnyı̆ AA. Clinico-

439 electroencephalographic correlations in asthenoadynamic subdepressions.Zh

440 Nevropatol Psikhiatr Im S S Korsakova. 1987;87(4):564-70. 
441 13.Lazarev VV. The relationship of theory and methodology in EEG studies of mental 442 activity.Int J Psychophysiol. 2006 Dec;62(3):384-93. Epub 2006 Mar 10.

443 14. Lazarev VV, Lebedeva IS, Tsutsul'kovskaia Mla, Severnyı̌ AA, Pozharitskaia DA. 444 Specificity of electroencephalographic correlations of asthenic disorders in atypical 445 juvenile asthenic-depressive conditions. Zh Nevropatol Psikhiatr Im S S Korsakova. $446 \quad 1990 ; 90(10): 73-7$.

447 15. Monakhov K, Perris C, Botskarev VK, von Knorring L, Nikiforov Al. Functional 448 interhemispheric differences in relation to various psychopathological components of the 449 depressive syndromes. A pilot international study.Neuropsychobiology. 1979;5(3):143$450 \quad 55$.

451 16. Schwarz E, Izmailov R, Spain M, Barnes A, Mapes JP, Guest PC, Rahmoune H, 452 Pietsch S, Leweke FM, Rothermundt M, Steiner J, Koethe D, Kranaster L, Ohrmann P, 453 Suslow T, Levin Y, Bogerts B, van Beveren NJ, McAllister G, Weber N, Niebuhr D, 454 Cowan D, Yolken $\mathrm{RH}$, Bahn S. Validation of a blood-based laboratory test to aid in the 455 confirmation of a diagnosis of schizophrenia. Biomark Insights. 2010 May 12;5:39-47.

456 17.Vigneau E, Qannari EM. Clustering of Variables Around Latent Components.

457 Communications in Statistics - Simulation and Computation. 2003; 32: 1131-1150.

458 18.Vigneau E, Chen M, Qannari E M. ClustVarLV: An R Package for the Clustering of 459 Variables Around Latent Variables. The R Journal. $2015 ; 7$ : 134-148. 
460

461

462

463

464

465

466

467

468 Feb;114(2):184-98.

469

470

471

472 in children with ADHD: an event-related potential study using the attention network test.

473 Int J Psychophysiol. 2011 Aug;81(2):82-90. doi: 10.1016/j.ijpsycho.2011.05.008.

474 24.Wechsler D. Wechsler Intelligence Scale for Children(WISC-III): Manual. 3th ed. San 475 Antonio: The Psychological Corporation; 1991.

476 25.Mello CB, Argollo Shayer PBM, Abreu N, Abreull N, Godinholll K, Durán P, Vargem

477 F, Muskat M, Miranda MC, Bueno OFA. Abbreviated version of the WISC-III: correlation 
478 between estimated IQ and global IQ of Brazilian children, Psicologia: Teor e Pesq. 2011; 47927 (2): 149-155. dx.doi.org/10.1590/S0102-37722011000200002.

480 26. Abramov DM, Pontes M, Pontes AT, Mourao-Junior CA, Vieira J, Quero Cunha C, 481 Tamborino T, Galhanone PR, deAzevedo LC, Lazarev VV. Visuospatial information 482 processing load and the ratio between parietal cue and target P3 amplitudes in the 483 Attentional Network Test. Neurosci Lett. 2017 Apr 24;647:91-96. doi:

484 10.1016/j.neulet.2017.03.031.

485 27.Gore Jr PA. Cluster Analysis, In: Tinsley HEA, Brown SD, editors. Handbook of 486 Applied Multivariate Statistics and Mathematical Modeling. San Diego, CA: Academic 487 Press; 2000. p. 297-321.

488 28. Landis JR, Koch GG. The measurement of observer agreement for categorical data. 489 Biometrics. 1977 Mar;33(1):159-74.

490 29. Petersen SE, Posner MI. The attention system of the human brain: 20 years after. 491 Annu Rev Neurosci. 2012;35:73-89. doi: 10.1146/annurev-neuro-062111-150525.

492 30. Kessler RC, Adler L, Ames M, Demler O, Faraone S, Hiripi E, Howes MJ, Jin R, 493 Secnik K, Spencer T, Ustun TB, Walters EE. The World Health Organization Adult 494 ADHD Self-Report Scale (ASRS): a short screening scale for use in the general 495 population. Psychol Med. 2005 Feb;35(2):245-56. 
496

497 Functional Asymmetry In The Central Brain Regions In Boys With Attention Deficit

498 Hyperactivity Disorder Detected By Event Related Potentials During Performance Of

499 The Attentional Network Test. 2016. Preprint.Avaiable on BioRxiv,

500 https://doi.org/10.1101/118380; cited on 06 July 2018.

501

502

503

504

505

506

507

508

509

510

511

512

513

514

32. Barkley RA. Behavioral inhibition, sustained attention, and executive functions:

constructing a unifying theory of ADHD. Psychol Bull. 1997 Jan;121(1):65-94. doi:

10.1037/0033-2909.121.1.65.

33. Barkley RA. Attention-Deficit/Hyperactivity Disorder: handbook for diagnosis and treatment. 3th ed. New York: The Guilford Press; 2006. p 32-33.

34. Langleben DD, Austin G, Krikorian G, Ridlehuber HW, Goris ML, Strauss HW.

Interhemispheric asymmetry of regional cerebral blood flow in prepubescent boys with

attention deficit hyperactivity disorder. Nucl Med Commun. 2001 Dec;22(12):1333-40.

35.Dang LC, Samanez-Larkin GR, Young JS, Cowan RL, Kessler RM, Zald DH.

Caudate asymmetry is related to attentional impulsivity and an objective measure of

ADHD-like attentional problems in healthy adults. Brain Struct Funct. 2016

Jan;221(1):277-86. doi: 10.1007/s00429-014-0906-6.

36. Lazarev VV, Pontes M, Pontes AT, Vieira J, Quero-Cunha C, Tamborino T, deAzevedo LC, Abramov DM. EEG and ERP characteristics of Attention Deficit 
515 Hyperactivity Disorder in children and adolescents [abstract]. Int. J. Psychophysiol. $516 \quad 2106 ; 108: 75$.

517 37. Vigneau E, Sahmer K, Qannari EM, Bertrand D. Clustering of variables to analyze 518 spectral data. J Chemometrics, 2005; 19: 122-128.

519 38. Vigneau E, Qannari EM, Navez B, Cottet V. Segmentation of consumers in 520 preference studies while setting aside atypical or irrelevant consumers. Food Quality 521 Pref. 2005; 47: 54-63.

522 39. Legland D, Beaugrand J. Automated clustering of lignocellulosic fibres based on 523 morphometric features and using clustering of variables. Industrial Crops and Products; $5242013 ; 45: 253-261$.

525 40. Lovaglio PG. Model building and estimation strategies for implementing the 526 Balanced Scorecard in Health sector. Quality \& Quantity. 2011; 45: 199-212.

527 41. Mackenzie GB, Wonders E. Rethinking Intelligence Quotient Exclusion Criteria

528 Practices in the Study of Attention Deficit Hyperactivity Disorder. Front Psychol. 2016 529 May 31;7:794. doi: 10.3389/fpsyg.2016.00794.

530 S1 table. Time intervals of Interest. Windows holding Cue-related potentials (CUE), 531 contingent voltage variation (CVV), and Target-related potentials (TGT) for each channel 
532 (ms), to calculate peak amplitude, peak latency, and mean amplitude. Zero ms refers to

533 Target onset (see Figure 1).

534 S2 method. Calculating the Kappa Index. Inference of the magnitude of agreement

535 between two categorical data (in this case, subjects' classification according to DSM and

536 to biological/behavioral variables in the dataset).

537 S3 dataset. DSM scores and variables of interest. In the first sheet: innatention,

538 hyperactive+impulsive, and total scores for ADHD according to DSM-IV-TR; second

539 sheet: all data concerning (1) scores of the WISC-III subtests, (2) ANT performance (AC:

540 accuracy, RT: reaction time and IVRT: intraindividual variation in reaction time, which is

541 the standard deviation of the subject's RT) for each experimental condition (Allcon:

542 mean value of all conditions, NtCue: neutral cue condition, SpCue: spatial cue condition,

543 ConTg: congruent target condition, IncTg: incongruent target condition) and attentional

544 network (alerting, orienting, and executive) plus learning indexes (IVRT or RT at the last

545 ANT block/first ANT block), and (3) the neurophysiological measures (peak amplitudes

546 and latencies, and mean amplitudes inside each time interval of interest in S1) for the

547 EEG channel set.

548 S4 output. DSM versus Biological agreement. The channel sets (rows) and number

549 of latent vectors extracted (rows) holding ERPs variables alone or ERPs + behavioral

550 (ANT and WISC) variables. The first block (on the left) are outputs from all 39 subjects. 
bioRxiv preprint doi: https://doi.org/10.1101/126433; this version posted September 28, 2018. The copyright holder for this preprint (which was not certified by peer review) is the author/funder. All rights reserved. No reuse allowed without permission.

551 The second block (on the right) are the averages of outputs with n-1 subjects (LOO

552 cross-validation). 


\section{Classifier Power}

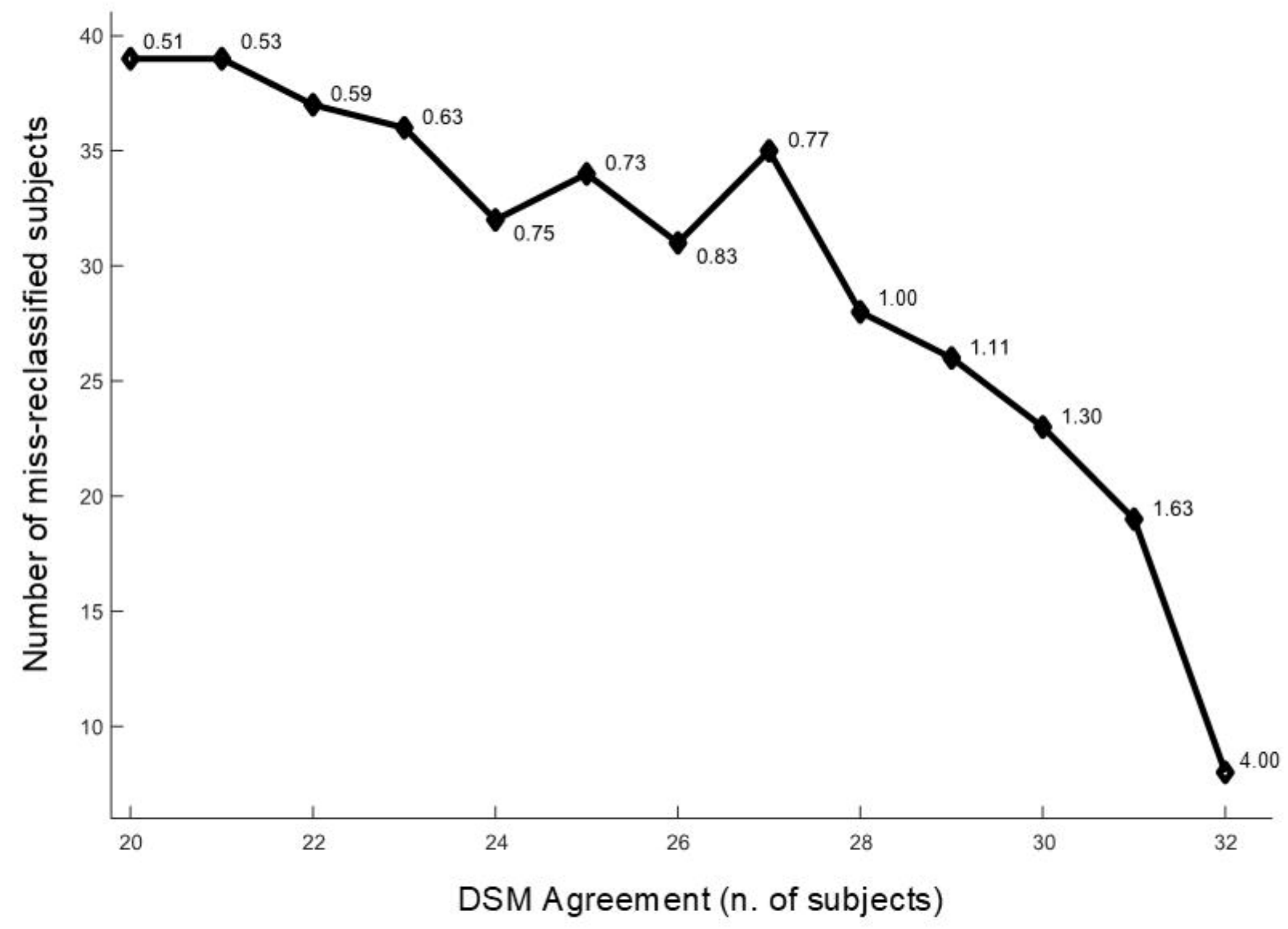

